\title{
Lieux de Travail Multiculturels et l'utilité des Modèles du Stress Professionnel Existants: Synthèse Bibliographique
}

\author{
Saoussany Amina, Enseignante chercheur \\ Asbayou Malika, Assistante de recherche en sciences de gestion \\ Equipe de Recherche en Gouvernance Information et Communication des \\ organisations, Université IBN ZOHR/ENCG Agadir, Maroc
}

Doi:10.19044/esj.2019.v15n25p182 URL:http://dx.doi.org/10.19044/esj.2019.v15n25p182

\section{Résumé}

Récemment, un nombre croissant d'études a été consacré à la recherche sur le stress en milieu professionnel et ses effets sur la santé des employés. Bien que les modèles conceptuels sur le stress aient tenté de saisir la plupart des aspects susceptibles d'influencer la santé et le bien-être des employés en milieu de travail, il est à noter cependant que le stress professionnel n'a pas encore été bien défini dans les contextes de travail multiculturels. L'objectif visé à travers la présente synthèse bibliographique est de passer en revue la littérature existante sur le stress au travail dans une perspective multiculturelle et d'analyser l'utilité des modèles existants dans ce nouveau contexte qui est jusqu'alors peu étudié. En effet, une recherche documentaire sur les bases de données scientifiques en ligne nous a permis de retenir cinq modèles ayant abordé le stress professionnel dans un contexte multiculturel. L'analyse de ces modèles fait ressortir qu'ils présentent des limites et doivent être élargis pour tenir compte des variables propres à ce nouveau contexte.

Mots clés: Pressions croissantes, Stress professionnel, Mondialisation, Lieux de travail multiculturels 


\title{
Multicultural Workplaces and the Utility of Existing Workplace Stress Models: Bibliographical summary
}

\author{
Saoussany Amina, Enseignante chercheur \\ Asbayou Malika, Assistante de recherche en sciences de gestion \\ Equipe de Recherche en Gouvernance Information et Communication des \\ organisations, Université IBN ZOHR/ENCG Agadir, Maroc
}

\begin{abstract}
Recently, an increasing number of studies have been devoted to occupational stress research and its effects on employees health. Although stress models have attempted to capture most of the aspects that can influence the health and well-being of employees in the workplace, it must be clear that work stress has not yet been clearly defined in multicultural work contexts. The purpose of this paper is to review the existing literature on occupational stress in a multicultural perspective and to evaluate the utility of existing models in this new context, which has been little studied. Indeed, a literature search on online scientific databases has identified five models that have addressed occupational stress in a multicultural context. Thus, the analysis of these models shows that they have limits and must be widened to take into account the variables specific to this new context.
\end{abstract}

Kewords: Increasing pressures, Occupational stress, Globalization, Multicultural workplaces

\section{Introduction}

La mondialisation des entreprises est une réalité qui définit la manière dont des personnes de nations et de cultures différentes travaillent ensemble. Plus de 63000 sociétés multinationales et globales et environ 821000 filiales étrangères emploient plus de 90 millions de personnes dans le monde selon Kuo (2011). Les Nations Unies estiment qu'environ 175 millions de personnes vivent en dehors de leur pays de naissance. Cette nouvelle ère a engendré des expériences stressantes pour les employés, notamment des pressions croissantes pour obtenir des résultats ainsi que des moyens de répondre aux exigences du marché mondial.

Depuis le début des années 90, la mondialisation a commencé à avoir un impact considérable sur les organisations. Pour les organisations des pays 
en développement, les impératifs de la mondialisation ne pouvaient plus être ignorés ou contournés. Pour ces entreprises, la création et le maintien d'avantages concurrentiels par divers moyens et formes sont devenus une préoccupation majeure (Salleh et al, 2008).

Ainsi, dans ce contexte de mondialisation et d'intensification de la concurrence, les employés de l'entreprise sont poussés à aller au-delà de leurs tâches habituelles. Les employés sont censés apprendre les différentes cultures, langues et règles du commerce international, ce qui se traduit par une charge de travail accrue, une pression accrue pour améliorer les compétences professionnelles et de longues heures de travail. De tels changements dans la nature de l'emploi, le milieu de travail et le comportement organisationnel augmenteraient sans aucun doute le stress professionnel des travailleurs, ce qui aurait une incidence sur leur santé physique et mentale (Salleh et al, 2008).

Plusieurs auteurs ont défini les sources de stress professionnel les plus courantes comme la charge et le rythme de travail, les conflits travail-famille, la responsabilité, les relations interpersonnelles (Mhiri, 2013) ou encore les longues heures de travail, le manque du soutien organisationnel (Davey et al, 2001). Or, aucun de ces facteurs n'inclue de problèmes spécifiques à un lieu de travail multiculturel. Bien que la plupart des cadres théoriques aient été proposés principalement pour analyser le stress professionnel sur la population considérée comme une majorité homogène, les relations entre les minorités ethniques et les facteurs de stress au travail ou alors les résultats pour la santé n'ont généralement pas été abordées. Or, avec la mondialisation du travail et la diversité croissante du lieu de travail, les modèles théoriques actuels de stress au travail doivent être adaptés pour inclure tous les groupes et tenter d'accroître la compréhension et le respect des différences entre ces groupes.

L'objectif de cet article est donc de réaliser une revue des modèles théoriques du stress professionnel existants et d'évaluer leur utilité pour les milieux de travail multiculturels. Ainsi, à travers une recherche documentaire, une revue de littérature du stress professionnel est dressée avant de s'intéresser à l'analyse des modèles théoriques retenus.

\section{Revue de littérature: le stress et le stress professionnel}

Le stress est un concept populaire qui est largement utilisé dans la vie quotidienne ainsi que dans diverses sciences de la vie. Cependant, comme souvent avec de tels concepts généraux, il existe des divergences considérables entre les différents domaines dans lesquels le concept est utilisé en ce qui concerne la définition et la connotation. Ainsi, cet article propose une brève évolution de cette notion avant de s'intéresser au stress professionnel. 


\section{Le stress : évolution d'un concept}

Etymologiquement, le terme stress provient du verbe latin stringere, qui veut dire bouleverser, déranger (Arnold et al., 1998).

Les premiers chercheurs qui ont étudié le stress ont adopté une approche physiologique. Par exemple, Hans Seyle définit le stress en 1956 comme "un syndrome général d'adaptation désignant un ensemble de réactions d'adaptation du corps à l'action spécifique de certains facteurs de stress et impliquant trois étapes: d'alarme, de résistance et d'épuisement".

Dans la phase d'alarme, des réactions spécifiques du corps se produisent lorsqu'un agent stressant est rencontré. Ainsi, l'immunité du corps diminue et si le facteur de stress est puissant la mort peut survenir. La phase de résistance représente l'état de mobilisation et de tension qui est ressentie comme stress. Les signes de réactions alarmantes disparaissent apparemment et la capacité de résistance dépasse le niveau normal. Au cours de cette phase, le corps utilise plus de ressources que la normale et l'épuisement des ressources représente la limite de la résistance lorsqu'il apparaît. Dans cette phase, les effets du stress peuvent être remarqués lorsqu'une détérioration de l'état de santé apparaît.

Scheuch (1996) estime que le stress est une activité humaine dont le but est de tenter de s'adapter aux changements intérieurs et extérieurs. Il définit le stress comme une réaction à un changement négatif des fonctions des organes et des changements qui se produisent entre une personne et son environnement social.

Lazarus et Folkman (1984) considèrent le stress comme le processus dans lequel l'individu joue le rôle d'agent actif pouvant influencer, aidé par des stratégies émotionnelles, cognitives et comportementales, les effets de la situation stressante. Ils définissent trois étapes dans l'évaluation du stress: l'évaluation primaire, l'évaluation secondaire et la réévaluation.

Dans l'évaluation primaire, l'appréciation de la situation a lieu. Il est établi à quel point une situation est dangereuse. L'évaluation secondaire fait référence aux possibilités d'adaptation et aux mesures que peut prendre l'individu pour faire face à la situation. La réévaluation fait référence à un changement de perception par rapport à l'évaluation principale.

Lazarus et Folkman ont adopté une vision du stress "interactionnelle" ou "transactionnelle". En d'autres termes, le stress représente la réaction des gens quand ils ne peuvent pas s'adapter aux exigences de l'environnement (familial, personnel ou au travail).

De nos jours, le stress est de plus en plus présent dans les milieux professionnels d'où l'apparition de la terminologie «stress professionnel » qu'il convient de définir. 


\section{Le stress professionnel}

Le stress est un état psychologique et physique qui résulte lorsque les ressources de l'individu ne sont pas suffisantes pour faire face aux exigences et aux pressions de la situation. Ainsi, le stress est plus probable dans certaines situations que dans d'autres et chez certaines personnes que d'autres (Michie, 2002).

Le stress professionnel peut être perçu comme la réaction d'un individu aux caractéristiques de l'environnement de travail qui sont perçues comme étant émotionnellement et physiquement menaçantes pour lui. Cela suppose une inadéquation entre les capacités de l'individu et son environnement de travail, où l'individu est soumis à des exigences excessives ou qu'il n'est pas tout à fait prêt à gérer la situation (Jamal, 1984; Ponnelle et al, 2012; Rascle et Irachabal, 2001). En général, plus le déséquilibre entre les demandes et les capacités des individus est élevé, plus le stress professionnel sera élevé (Jamal, 2007).

Conformément à la conceptualisation du stress de Lazarus (1966), le stress professionnel ne réside ni dans l'environnement de travail ni dans l'individu. C'est au contraire l'évaluation cognitive de la situation au sein de l'environnement de travail qui est au cœur de ce processus de stress organisationnel.

Beehr et Newman (1978), après un examen approfondi d'une sélection de recherches sur le stress, définissent le stress professionnel comme une condition dans laquelle les facteurs liés au travail interagissent avec l'individu pour modifier (perturber ou améliorer) son état psychologique ou physiologique de telle sorte que le corps ou l'esprit de la personne est forcé de s'écarter du fonctionnement normal. Beehr et Newman suggèrent que le stress provient de conditions pouvant être considérées comme positives (amélioration) ou négatives (perturbation).

Cooper et Marshall (1976) indiquent que par stress professionnel, on entend des facteurs environnementaux négatifs ou des facteurs de stress (surcharge de travail, conflit de rôle/ ambiguïté, mauvaises conditions de travail, par exemple) associés à un travail particulier.

Il ressort de ces définitions communes du stress que dans la plupart des recherches sur le stress en organisation et en psychologie industrielle, les facteurs organisationnels et les individuels ont été utilisés pour définir le stress (Cooper \& Marshall, 1976; McGrath, 1976; Beehr \& Newman, 1978). En d'autres termes, le stress est une relation personne-environnement ou une interaction des caractéristiques de la personne et des sources potentielles de stress dans le milieu de travail.

Le stress professionnel peut donc être défini par les caractéristiques individuelles de la personne concernée comme par l'ampleur des facteurs organisationnels auxquels l'individu est exposé dans l'exercice quotidien de 
son activité. Les lieux de travail multiculturels constituent un champ d'étude intéressant afin de mieux comprendre le phénomène du stress à l'ère de la mondialisation.

\section{Evaluation de l'utilité des modèles du stress professionnel pour les lieux de travail multiculturels}

Dans le but de mieux expliquer le stress au travail et ses conséquences sur la santé et le bien-être individuels, cinq modèles principaux de stress ont été examinés dans la littérature. Ils ont été utilisés pour mieux comprendre les relations complexes entre les facteurs de stress au travail et les influences négatives et / ou positives qui en résultent sur la santé. Le premier point de cet axe sera dédié à la présentation des modèles retenus.

\section{Présentation des modèles du stress professionnel retenus}

Suite à une recherche documentaire sur les base des données scientifiques en ligne ( Elsevier, Cairn, HAL archives ouvertes, Jstore, semanticscholar, google scholar, sciencedirect et l'outil de recherche documentaire sur le logiciel de gestion bibliographique Mendeley) en utilisant comme mots clés: occupational stress, stress and globalization et multicultural workplace stress, une centaine d'articles a été collectée et analysée pour retenir cinq grands modèles sur le stress professionnel dans ce contexte multiculturel comme illustré dans le tableau 1.

Tableau 1: les modèles du stress professionnel retenus

\begin{tabular}{|l|l|l|}
\hline Modèle & Fondateur & Année \\
\hline Le modèle de contrôle- demande-soutien & Karasek & 1980 \\
\hline Le modèle du déséquilibre efforts-récompenses & Siegrist & 1996 \\
\hline Le modèle d'ajustement personne-environnement & Caplan et Harrison & 1993 \\
\hline La théorie de l'écologie sociale & Stokols & 1992 \\
\hline La théorie laïque du stress & Furnham & 1997 \\
\hline
\end{tabular}

Depuis les années 1980, le principal modèle de stress professionnel, en psychologie de la santé au travail, est le modèle de contrôle de la demande proposé par Karasek (1981). Le modèle suppose essentiellement que la combinaison d'un contrôle faible et d'une demande élevée conduit à des résultats négatifs pour la santé. En d'autres termes, les employés qui font face à des exigences élevées sur le lieu de travail et ont peu de contrôle sur leur travail sont considérés comme exposés à des facteurs de stress susceptibles d'avoir une influence négative sur leur santé (Grebner et al, 2005 ; Ganster et al, 2001).

Le soutien social au travail, une composante négligée, a ensuite été intégré au modèle; en conséquence, le modèle contrôle-demande-soutien a été défini. Ce nouveau modèle suppose que le risque de maladie le plus élevé est attendu chez les employés à forte demande, à faible contrôle et à faible soutien 
social sur le lieu de travail. Bien que ce modèle soit très similaire au modèle de contrôle de la demande, le premier souligne l'hypothèse selon laquelle les pressions psychologiques et sociales subies par les personnes sur le lieu de travail résultent de relations sociales et interpersonnelles entre les participants au processus de travail (Helland Hammer, 2004 ; Polanyi et Tompa, 2004).

Le modèle de déséquilibre effort-récompense sur le lieu de travail, proposé par Siegrist (1996), représente un cadre théorique différent utilisé pour définir le stress lié au travail. Ce modèle postule que le déséquilibre entre les efforts déployés sur le lieu de travail et la récompense professionnelle conduit à une détresse et à des effets néfastes sur la santé (Van Vegchel et al, 2005). Le modèle de Siegrist insiste sur le fait que le rôle professionnel peut offrir des possibilités d'expérience positive, d'augmentation de l'autoefficacité et d'expérience positive récurrente d'estime de soi, s'il est suffisamment récompensé. Inversement, le fait que les employeurs ne reconnaissent pas ou ne récompensent pas leurs efforts peut être préjudiciable à la santé et au bien-être des employés (Rugulies et al., 2009).

Un autre modèle théorique largement utilisé dans la recherche est celui d'ajustement personne - environnement $(\mathrm{P}-\mathrm{E})$ proposé par Caplan et Harrison (1993). Ce modèle caractérise le stress comme un manque de correspondance entre les caractéristiques de la personne et l'environnement de travail (Elovainio, 2000). Essentiellement, le concept d'ajustement $\mathrm{P}-\mathrm{E}$ suggère que l'alignement entre les caractéristiques des personnes et les environnements de travail aboutit à des résultats positifs pour les individus et les organisations. Inversement, un grand nombre de recherches (Ettner et Grzywacz, 2001) montre qu'un mauvais ajustement entre les caractéristiques individuelles et l'environnement de travail, en particulier chez les personnes qui ont tendance à réagir au stress avec des états émotionnels négatifs, augmente le risque de problèmes de santé.

La théorie de l'écologie sociale proposée par Stokols (1992) représente un autre modèle utilisé dans la recherche sur le stress professionnel. Cette théorie met l'accent à la fois sur la personne et sur l'environnement et suggère que les dispositions, ressources et caractéristiques individuelles façonnent les perceptions de la manière dont l'emploi influence la santé (Ettner et Grzywacz, 2001). Ainsi, afin de déterminer comment un travail et / ou un environnement de travail spécifique influence les résultats pour la santé, il convient de contrôler les attributs individuels susceptibles de fausser la notification des effets des tâches sur la santé. Par exemple, les travailleurs qui présentaient des niveaux plus élevés de contraintes perçues et de névrotisme, qui travaillaient la nuit ou des heures supplémentaires, ou qui signalaient un stress grave et continu au travail ou une pression professionnelle supérieure, ont signalé des résultats négatifs pour la santé (Ettner et Grzywacz, 2001). 
Enfin, une théorie laïque du stress a été proposée par Furnham (1997). Cette théorie utilise une représentation laïque du stress et représente une approche différente dans la tentative de délimiter les sources de stress professionnel. En tant que telle, cette théorie est conceptualisée comme l'explication de bon sens que les gens fournissent aux aspects du comportement social. Certains chercheurs [par exemple : (Kinman et Jones, 2005)] soutiennent que la connaissance de la manière dont les individus interprètent le concept de stress est particulièrement utile pour comprendre les manifestations de stress professionnel interculturelles. Par exemple, en raison de différences culturelles, les gens pourraient décrire la maladie différemment et présenter même des symptômes différents. Reconnaître que des personnes de groupes culturels différents peuvent vivre ou exprimer le stress professionnel différemment serait très utile pour conceptualiser le stress professionnel en fonction de la culture.

\section{Evaluation de l'utilité des modèles du stress professionnel retenus:}

La littérature analysée souligne que la majorité des recherches empiriques existantes ne prennent pas spécifiquement en compte les caractéristiques des lieux de travail multiculturels lorsqu'on analyse le stress lié au travail. Très peu de modèles théoriques utilisés pour étudier le stress au travail se sont concentrés sur l'établissement de relations entre les caractéristiques / facteurs de stress professionnels et les caractéristiques individuelles, telles que le développement personnel, l'adaptation et le changement, tout en tenant compte de diverses caractéristiques et origines culturelles. La revue précédente de la littérature a mis en évidence trois thèmes concernant les modèles de stress existants à utiliser dans un lieu de travail multiculturel.

Premièrement, les deux modèles dominants reliant le travail et la santé sont le modèle de contrôle de la demande d'emploi et le modèle de déséquilibre effort-récompense. L'utilisation de ces deux modèles dans les études actuelles a généralement montré, toutefois, que certains aspects des expériences de travail, résultant des nouvelles réalités du lieu de travail, vont au-delà des concepts de base des modèles. Par conséquent, ces modèles doivent être réexaminés pour les recherches futures, leur permettant de saisir toute la gamme des expériences des travailleurs dans les lieux de travail de plus en plus flexibles, multiculturels et compétitifs d'aujourd'hui.

Deuxièmement, la majorité des études existantes analysant le sujet du stress lié au travail n'explorent que la surface des effets d'interaction au sein des groupes interculturels et entre ceux-ci. Tous les modèles théoriques discutés ci-dessus ont été utilisés pour expliquer le stress lié au travail; Cependant, la plupart d'entre eux ont omis toute considération et applicabilité interculturelles. 
Troisièmement, la littérature semble suggérer qu'un soutien social sur le lieu de travail peut être très utile pour faciliter le processus général d'adaptation des employés dans des lieux de travail multiculturels. Ainsi, l'impact du soutien social en général et du soutien en milieu de travail en particulier sur la santé des employés devrait être considéré comme extrêmement important. Les recherches futures devraient tenter de modifier les modèles théoriques existants pour inclure de nouvelles configurations d'environnement social et professionnel. De telles recherches devraient également tenter de démontrer que le soutien social et professionnel est étroitement lié aux caractéristiques personnelles d'un individu et que, par conséquent, ces facteurs doivent jouer un rôle plus spécifique dans la recherche.

En conclusion, du point de vue interculturel, chacun de ces modèles fait défaut. Autrement dit, pour les modèles de stress professionnel les plus cités, l'accent est mis sur la manière dont le travailleur interagit avec et / ou interprète l'environnement de travail. En général, ces modèles souvent cités ne reconnaissent pratiquement pas l'influence substantielle des facteurs sociétaux et interpersonnels liés au processus d'intégration. Par exemple, l'objectif principal de nombreuses études utilisant ces modèles a été d'identifier les sources de stress au travail les plus courantes.

Dans ce sens, Tous les modèles théoriques présentés ci-dessus se concentrent sur divers aspects du travail et de l'environnement de travail susceptibles d'avoir une influence sur la santé et le bien-être individuels. Compte tenu de l'évolution du monde du travail multiculturel, de nombreuses voix de ce domaine de recherche reconnaissent la nécessité de repenser et / ou d'élargir ces modèles théoriques afin de saisir toutes les dimensions du travail qui pourraient, directement ou indirectement, affecter la santé des employés (Polanyi et Tompa, 2004). En particulier, il est absolument nécessaire d'identifier les dimensions contemporaines du travail qui pourraient influer sur la qualité de l'expérience de travail dans les lieux de travail multiculturels d'aujourd'hui. Cela peut être rendu possible par une exploration attentive de tous les facteurs du lieu de travail susceptibles de modifier la relation entre les intérêts et les responsabilités liées au travail, à la famille et à la vie. Par exemple, la plupart des études sur le stress au travail s'accordent pour dire que les dimensions essentielles du travail susceptibles de modifier la manière dont les expériences de travail sont perçues par les employés sont les suivantes: exigences du travail et latitude décisionnelle, qualité des interactions sociales, organisation du travail et adéquation entre les intérêts des travailleurs et leurs emplois (Polanyi et Tompa, 2004 ; Ettner et Grzywacz, 2001 ; Beiser, 1999 ; Wang et Patten, 2001). 


\section{Conclusion}

La majorité des études examinées dans la présente communication ne modélisaient pas consciemment les interactions entre les facteurs personnels et professionnels et ne tenaient pas spécifiquement compte des caractéristiques multiculturelles du lieu de travail lors de l'analyse du stress lié au travail. En outre, le débat sur le point de savoir si les personnes de différents groupes culturels vivent ou expriment différemment le stress lié au travail représente un domaine important de recherche en cours. Par exemple, l'interprétation et la représentation personnelles du stress sur le lieu de travail sont susceptibles de façonner les perceptions et l'expérience des individus concernant l'influence positive ou négative du travail sur la santé et, en outre, de déterminer le type de mesures correctives à prendre.

Tenant compte du fait que la diversité de la main-d'œuvre est en train de devenir l'un des problèmes les plus urgents de la vie organisationnelle, les recherches futures devraient utiliser de nouvelles approches afin de combler les lacunes dans la compréhension de la dynamique de la diversité et de ses implications pour la recherche sur le stress professionnel. L'accent principal pourrait être mis sur le point de savoir si les conclusions concernant l'influence de l'environnement de travail sur les attitudes et le comportement des employés au travail s'appliquent de la même manière à la diversité multiculturelle de la population active actuelle.

\section{References:}

1. Arnold, J., Cooper, C.L., and Robertson, I.T. (1998). Work Psychology: Understanding Human Behaviour in the Workplace. Editura Pitman, Londra

2. Beehr, T.A., Newman, J.E. (1978). Job Stress, Employee Health And Organizational Effectiveness: A Facet Analysis, Model And Literature Review. Personnel Psychology, 31, 665-699

3. Beiser M (1999). The health of immigrants and refugees in Canada. Canadian Journal of Public Health ,96, 30-44

4. Caplan RD, Harrison RV (1993). Person-environment fit theory: some history, recent developments, and future directions. J Soc Issues, 49, 253-276

5. Cooper, C. L., \& Marshall, J. (1976). Occupational sources of stress: A review of the literature relating to coronary heart disease and mental ill health. Journal of Occupational Psychology, 49, 11-28

6. Davey, J. Obst, P. \& M. Sheehan M. (2001). Demographic and workplace characteristics which add to the prediction of stress and job satisfaction within the police workplace, Journal of Police and Criminal Psychology, 16(1), 29-39 
7. Elovainio M, (2000). Organizational and individual factors affecting mental health and job satisfaction: a multilevel analysis of job control and personality. J Occup Health Psychology, 5, 269-277

8. Ettner SL, Grzywacz JG (2001). Workers' perceptions of how drugs affect health: a social ecological perspective. Journal of Occupational Health Psychology, 6, 101-113.

9. Furnham A (1997). Lay theories of work stress. Work Stress, 11, 6878

10. Ganster DC, Dewyer DJ, Fox ML (2001). Explaining employees' healthcare costs: a prospective examination of stressful job demands, personal control, and physiological reactivity. Journal of Applied Psycholgy ,86, 954-64

11. Grebner S, Semmer NK, Elfering A (2005). Working conditions and three types of well-being: a longitudinal study with self-report and reading data. Journal of Occupational Health Psychology, 10, 31-43

12. Helland Hammer TH, (2004). Expanding the psychosocial work environment: workplace norms and work-family conflict a scoreless stress and health. Journal of Occupational Health Psychology, 9, 8397.

13. Jamal M. (1984). Job Stress and job Performance controversy: an empirical assessment in two countries. Organizational Behaviour and Human Performance, 33,1-21.

14. Jamal, M. (2007). Job stress and job performance controversy revisited: An empirical examination in two countries. International Journal of Stress Management, 14(2), 175- 187

15. Karasek RA, Baker D, Marxer F, Ahlborn A, Theorell T (1981). Job decision latitude, job demands and cardiovascular disease: a prospective study of Swedish men. Am J Public Health, 71, 694-705

16. Kinman G, Jones F (2005). Lay representations of workplace stress: what do people really mean when they say that they are stressed. Work Stress , 19, 101-120

17. Kuo, B.C.H. (2011). Cultural variations in work stress and coping in an era of globalization. Camabridge Handbook of Culture, Organizations, \& Work, 418-441

18. Lazarus, R. S. (1966). Psychological stress and the coping strategy, New York: Mc Graw-Hil

19. Lazarus R. S. \& Folkman S. (1984), Stress, Appraisal, and Coping, Springer Publishing Company.

20. McGrath , J. E. (1976) . Stress and behavior in organizations. in M. D. Dunnette (ed.), Handbook of Industrial and Organizational Psychology . Chicago, IL : Rand McNally 
21. Mhiri, S. (2013). L'impact du stress professionnel sur l'implication organisationnelle, l'absentéisme et l'intention de quitter chez les cadres, thèse de doctorat en sciences de gestion.

22. Michie S., (2002). Causes and management of stress at work. Occupational and Environmental Medicine , 59(1), 67-72

23. Polanyi M, Tompa E. (2004). Rethinking work-health models for the new global economy: a qualitative analysis of emerging dimensions of work. Work,23, 3-18

24. Ponnelle S., Vaxevanoglou X., \& Garcia F., (2012). L'usage des outils d'évaluation du stress au travail : perspectives théoriques et méthodologiques. Le travail humain, 2(75), 179-213.

25. Rascle N., \& Irachabal S., (2001). Médiateurs et modérateurs : implications théoriques et méthodologiques dans le domaine du stress et de la psychologie de la santé. Le travail humain, 64(20), 97-118.

26. Rugulies R, et al. (2009). Distribution of effort-reward imbalance in Denmark and its prospective association with a decline in selfrated health. Journal of Occupational Environment Med, 51, 870-878.

27. Salleh, A. L., Bakar, R. A. \& Keong W. K., (2008). How Detrimental is Job Stress? : A Case Study Of Executives in the Malaysian Furniture Industry. International Review of Business Research Papers Vol. 4(5), 64-73

28. Sauter SL, Murphy LR, Hurrell JJ (1990). Prevention of work-related psychological disorders: a national strategy opposed by the National Institute for Occupational Safety and Health. Am Psychol,45, 11461158.

29. Seigrist J (1996). Adverse health effects of high-effort/low-reward conditions. Journal of Occupational Health Psychology ,1, 27-41.

30. Selye, H (1956). The Stress of life. New York : McGraw-Hill.

31. Stokols D (1992). Establishing and maintaining healthy environments: toward a social ecology of health promotion. Am Psychology,47, 6-22

32. Van Vegchel N, De Jonge J, Landsbergis PA (2005). Occupational in (inter)action: the interplay between job demands and job resources. Journal of Organizational Behavior , 26, 535-560.

33. Wang J, Patten SB (2001). Perceived work stress and major depression in the Canadian employed population, 20-49 years old. Journal of Occupational Health Psychology, 6, 283-289. 